\title{
CONNECTION BETWEEN THE LEAKING AND THE VISCOELASTIC BEHAVIOR OF FLANGE GASKETS
}

\author{
GÁbor L. Szepesi - Zoltán Siménfalvi \\ Institute of Energy Engineering and Chemical Machinery, Dept. of Chemical Machinery \\ University of Miskolc \\ H-3515 Miskolc-Egyetemváros, Hungary \\ szepesi@uni-miskolc.hu
}

[Received: October 20, 2014, Accepted: February 12, 2015.]

\begin{abstract}
This paper presents a measurement and calculation method to determine the stress relaxation function parameters of a flange gasket which has a viscoelastic behavior. It is so important, because it has a strong connection to the vessels leakage.
\end{abstract}

Mathematical Subject Classification: 05C38, 15A15

Keywords: gasket, stress relaxation, Generalized Maxwel Model, penalty function.

\section{INTRODUCTION}

Operation of closed systems often cause isolation problems. In this case the air contaminant may leak into the working area or into the environment. The flange-gasket untightness is the source of the leaking most times. This paper points out the main cause of the leakage of soft PTFE (Polytetrafluoroethylene) covered textile gasket between flange joints. An investigation unit has been created to examine the PTFE covered gaskets. With the help of this investigation unit, the stress and deformation in the gasket can be measured.

\section{Gasket investigation Unit}

The investigation unit has been created for gasket measuring is shown in Figure 1 . The main parts of the investigation unit are:

1. tension tester (load capacity: $25 \mathrm{kN}$ ),

2. load cell,

3. flange,

4. gasket,

5. displacement transmitter,

6. A/D converter,

7. computer. 
During the measurements the flange gasket is pressed by the tension tester. The compression-stress and the gasket deformation (compressive strain) recorded by the A/D logger-converter. When the stress reaches the maximum, the increment of the stress is stopped. With this procedure we can simulate a flange-joint gasket deformation and stress relaxation.

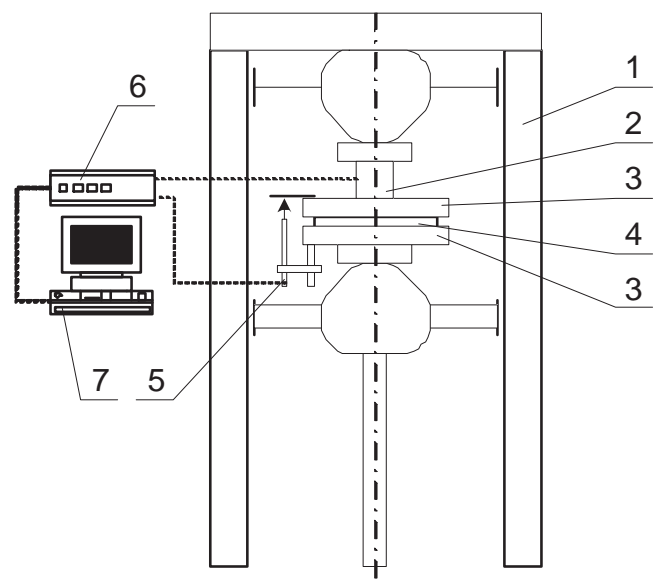

Figure 1. The investigation unit

If the gasket is not working properly leaking can occur. This happens if the gasket parameters are not correct or the gasket is damaged. If the gasket stress can not reach the required value or the stress is reduced below the required value a leaking process can start. Due to the leakage, the air contaminant mass flow spilling into the atmosphere is determinable[1].

\section{Mechanical model of Flange CONNECtion}

The simplified mechanical model of flange connection is showed on Figure 2. The base load of the flange is the bending momentum. This load arise from the bolt force, the inner pressure force and the gasket force. The flange and the gasket forces are different in case of operation state and assembling state. The inner pressure forces are zero in case of assembling state. In the present case the gasket force is higher then another state. The minimum bolt force in assembling state can calculate by:

$$
W_{A}=\pi b G y
$$

where $b$ is the effective gasket width, $G$ is the diameter of the gasket center line, $y$ is the minimal gasket stress.

If the applied bolt force is lower than $W_{A}$ (calculated with (1)) the gasket is not working acceptably and it cause leaking. 
In case of operational state the bolt force has to be higher then assembling state. This force can calculate with this equation:

$$
W_{O P}=\frac{\pi}{4} G^{2} P+2 \pi G m P,
$$

where $P$ is the pressure, $m$ is the gasket parameter. This gasket parameter depends on the material of the gasket. The table shows the gasket parameter and the minimal gasket stress in case of different type of gaskets:

Table 1. Typical gasket parameters and minimal stresses

\begin{tabular}{|c|c|c|}
\hline Type of the gasket & Gasket parameter, $m$ & Gasket minimal stress, $y, M P a$ \\
\hline Rubber & $0.5-1$ & $0-1.4$ \\
PVC & 1.5 & 1.2 \\
PTFE & $2-2.75$ & $1.2-1.6$ \\
Rubber with textile & 1.25 & 2.75 \\
IT sheet & $2.25-2.75$ & $15-25$ \\
Wawed seal & $2.5-3.5$ & $25-52$ \\
\hline
\end{tabular}

The effective gasket stress is depends on the bolt force, the gasket parameter, the gasket minimal stress and of course the geometry of the flanged connection. If

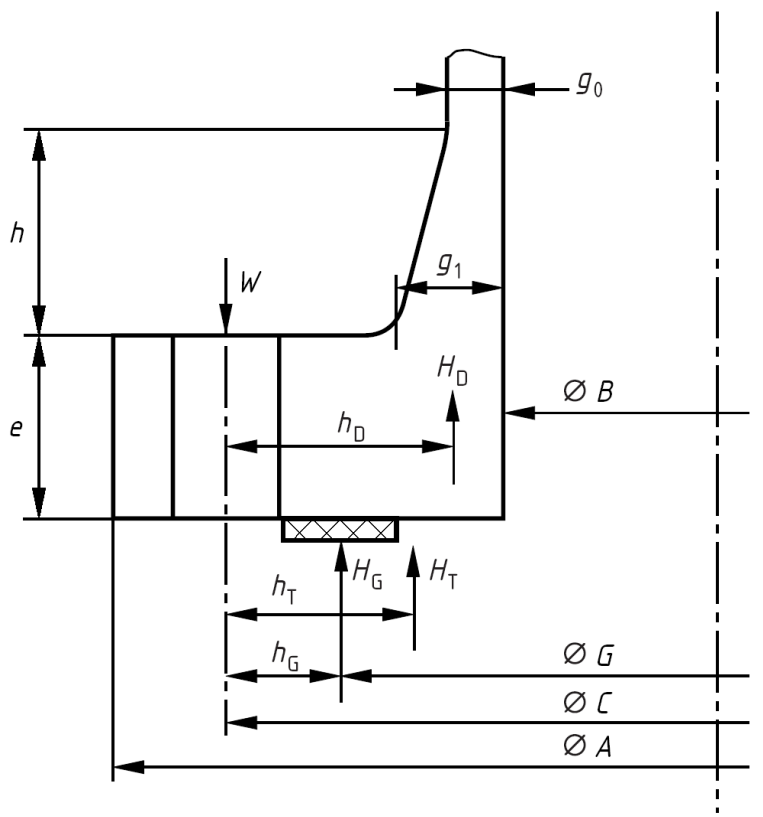

Figure 2. General mechanical model of flange connection 
the gasket material shows viscoelastic or viscoplastic property, the gasket stress also depends on the time.

\section{Generalized Maxwell Model}

The material of the PTFE covered textile gasket shows viscoelastic property. The viscoelastic material-model is described by rheological elements. The Generalized Maxwell model[2], shown in Figure 3 is used for describing the material behavior of the gasket.

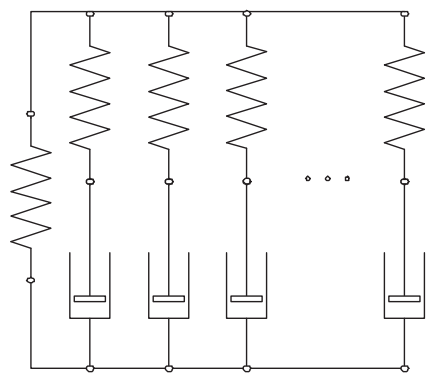

Figure 3. Generalized Maxwell Model

Assuming that gasket deformation is only in axial direction, there is no radial deformation. Consequently, only volumetric stresses occur in the gasket. This linear viscoelastic behavior is commonly using the Boltzmann superposition integral[4]:

$$
\sigma(\tau)=\int_{0}^{\tau} K\left(\tau-\tau^{\prime}\right) \frac{\partial \epsilon}{\partial \tau^{\prime}} \partial \tau^{\prime}
$$

where $K$ is the relaxation function, $\tau$ is the time, $\epsilon$ is the deformation. The relaxation function is approximated with the following formula:

$$
K(\tau)=K_{\infty}+K_{0} \sum_{k=1}^{m} w_{k} e^{-\frac{\tau}{\tau_{k}}} .
$$

The $\sigma(\tau)$ stress-function approximated with:

$$
f_{k}(t)=A+B \sum_{j=1}^{m} w_{j} e^{-t / \tau_{k}},
$$

where $A$ is the residual stress, $B$ is the relaxation factor, $w_{j}$ is the weighting coefficient, $m$ is the number of the Maxwell elements, $\tau_{k}$ is the relaxation time of the one of the Maxwell element.

According to the investigation results, in case of $m=3$, the approximation is suitable. The least squares method is used in the approximation process: 


$$
F=\sum_{i=1}^{n}\left(f_{k i}-f_{m i}\right)^{2} \rightarrow \min ,
$$

where $n$ is the number of the measuring points, $f_{k i}$ the approximated stressfunction, $f_{m i}$ is the measured stress values.

Derivative of function 6 with respect to the variable $A$ :

$$
\frac{\partial F}{\partial A}=2 \sum_{i=1}^{n}\left(f_{m i}-f_{k i}\right) .
$$

Derivative of function $(6)$ with respect to the variable $B$ :

$$
\frac{\partial F}{\partial B}=2 \sum_{i=1}^{n}\left(f_{m i}-f_{k i}\right) \cdot \sum_{j=1}^{m}\left(w_{j} e^{-\frac{t_{i}}{\tau_{j}}}\right) .
$$

Derivative of function (6) with respect to the variable $w_{k}$, where $\mathrm{k}=1,2,3$ :

$$
\frac{\partial F}{\partial w_{k}}=2 \sum_{i=1}^{n}\left(f_{m i}-f_{k i}\right)\left[B e^{-t_{i} / \tau_{k}}\right] .
$$

Derivative of function (6) with respect to the variable $\tau_{k}$, where $\mathrm{k}=1,2,3$ :

$$
\frac{\partial F}{\partial \tau_{k}}=2 \sum_{i=1}^{n}\left(f_{m i}-f_{k i}\right)\left[B w_{k} \frac{t_{i}}{\tau_{k}^{2}} e^{-t_{i} / \tau_{k}}\right] .
$$

The eight nonlinear equation involves eight unknown parameters. These parameters give to the approximation-function unknown values. The nonlinear equation systems in reduced form is the following:

$$
\begin{gathered}
\sum_{i=1}^{n}\left[A+B\left(w_{1} e^{-\frac{t_{i}}{\tau_{1}}}+w_{2} e^{-\frac{t_{i}}{\tau_{2}}}+w_{3} e^{-\frac{t_{i}}{\tau_{3}}}\right)-f_{m i}\right]=0 \\
\sum_{i=1}^{n}\left[A+B\left(w_{1} e^{-\frac{t_{i}}{\tau_{1}}}+w_{2} e^{-\frac{t_{i}}{\tau_{2}}}+w_{3} e^{-\frac{t_{i}}{\tau_{3}}}\right)-f_{m i}\right]\left[w_{1} e^{-\frac{t_{i}}{\tau_{1}}}+w_{2} e^{-\frac{t_{i}}{\tau_{2}}}+w_{3} e^{-\frac{t_{i}}{\tau_{3}}}\right]=0 \\
\sum_{i=1}^{n}\left[A+B\left(w_{1} e^{-\frac{t_{i}}{\tau_{1}}}+w_{2} e^{-\frac{t_{i}}{\tau_{2}}}+w_{3} e^{-\frac{t_{i}}{\tau_{3}}}\right)-f_{m i}\right]\left[B e^{-\frac{t_{i}}{\tau_{1}}}\right]=0 \\
\sum_{i=1}^{n}\left[A+B\left(w_{1} e^{-\frac{t_{i}}{\tau_{1}}}+w_{2} e^{-\frac{t_{i}}{\tau_{2}}}+w_{3} e^{-\frac{t_{i}}{\tau_{3}}}\right)-f_{m i}\right]\left[B e^{-\frac{t_{i}}{\tau_{2}}}\right]=0
\end{gathered}
$$




$$
\begin{aligned}
& \sum_{i=1}^{n}\left[A+B\left(w_{1} e^{-\frac{t_{i}}{\tau_{1}}}+w_{2} e^{-\frac{t_{i}}{\tau_{2}}}+w_{3} e^{-\frac{t_{i}}{\tau_{3}}}\right)-f_{m i}\right]\left[B e^{-\frac{t_{i}}{\tau_{3}}}\right]=0 . \\
& \sum_{i=1}^{n}\left[A+B\left(w_{1} e^{-\frac{t_{i}}{\tau_{1}}}+w_{2} e^{-\frac{t_{i}}{\tau_{2}}}+w_{3} e^{-\frac{t_{i}}{\tau_{3}}}\right)-f_{m i}\right]\left[B w_{1} \frac{t_{i}}{\tau_{1}^{2}}\right]=0 . \\
& \sum_{i=1}^{n}\left[A+B\left(w_{1} e^{-\frac{t_{i}}{\tau_{1}}}+w_{2} e^{-\frac{t_{i}}{\tau_{2}}}+w_{3} e^{-\frac{t_{i}}{\tau_{3}}}\right)-f_{m i}\right]\left[B w_{2} \frac{t_{i}}{\tau_{2}^{2}}\right]=0 . \\
& \sum_{i=1}^{n}\left[A+B\left(w_{1} e^{-\frac{t_{i}}{\tau_{1}}}+w_{2} e^{-\frac{t_{i}}{\tau_{2}}}+w_{3} e^{-\frac{t_{i}}{\tau_{3}}}\right)-f_{m i}\right]\left[B w_{3} \frac{t_{i}}{\tau_{3}^{2}}\right]=0 .
\end{aligned}
$$

If this equation system is solved, we get the approximation-functions' parameters. During this minimization method, the following equations should be satisfied:

$$
\begin{aligned}
& \sum_{j=1}^{k} w_{k}-1=0 \rightarrow h(X)=0 \\
& {\left[\begin{array}{c}
-A \\
-B \\
-w_{1} \\
-w_{2} \\
-w_{3} \\
\tau_{1} \\
\tau_{2} \\
\tau_{3}
\end{array}\right] \leq 0 \rightarrow g(X) \leq 0 .}
\end{aligned}
$$

The following constrained-extremum problem should be solved in order to simplify:

$$
\begin{gathered}
F(X) \rightarrow \min \\
h(X)=0, \\
g(X) \leq 0 .
\end{gathered}
$$

Relevant mathematical literature offers a lot of methods to solve (21). A penaltyfunction technique [3] is used to solve the problem. The following penalty function is used in the procedure:

$$
\Theta(X, \sigma)=F(X)+\sigma \sum_{q=1}^{r} h_{q}^{2}(X)+\sigma \sum_{y=1}^{c}\left(\max \left(g_{y}(X), 0\right)\right)^{2} .
$$

The constrained-extremum problem (21) can be converted to an unconditional extremum problem with the help of the penalty function. The Nelder-Mead procedure, which is implemented in MATLAB, is used to solve the problem. For the $\sigma$ sequence: $\sigma_{k}=10^{k-1}$. 
Figure 4 shows one of the approximated results.

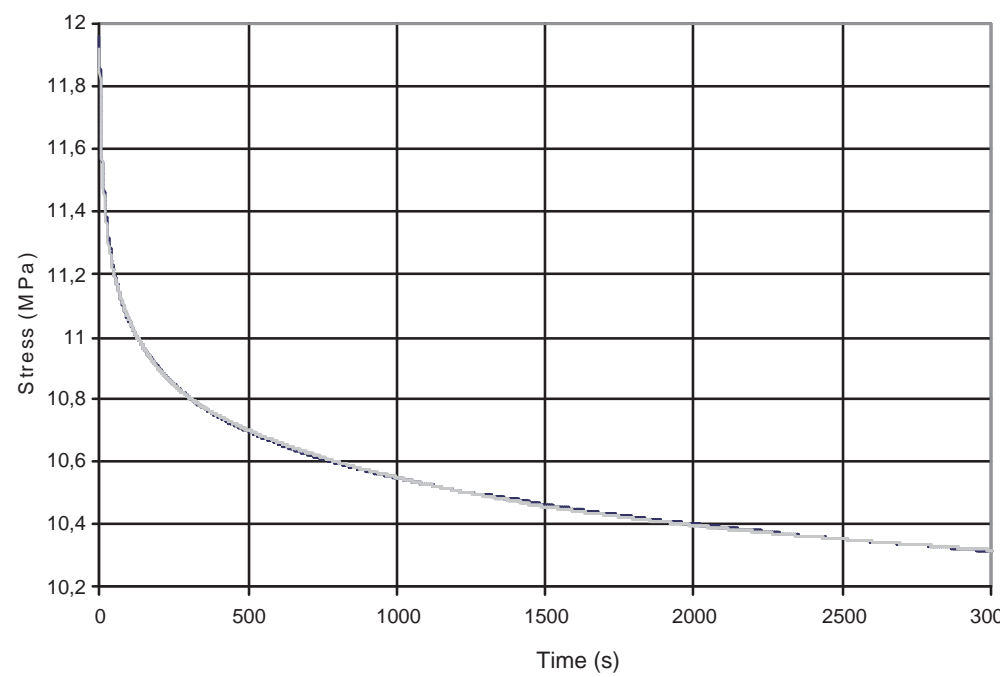

Figure 4. The measured and calculated stress

Measurements are made in different maximal gasket stress states. Summary of the approximation results are shown in the tables.

Results for $3 \mathrm{MPa}$ gasket loading:

\begin{tabular}{|c|c|c|c|c|c|c|c|c|c|}
\hline No. & $A$ & $B$ & $w_{1}$ & $w_{1}$ & $w_{1}$ & $\tau_{1}$ & $\tau_{1}$ & $\tau_{1}$ & $A / \sigma_{\max }$ \\
\hline \hline 1 & 2.03 & 0.58 & 0.37 & 0.28 & 0.35 & 19.9 & 556 & 7454 & 0.77 \\
2 & 1.94 & 0.49 & 0.35 & 0.27 & 0.38 & 40.1 & 634 & 7388 & 0.77 \\
3 & 1.96 & 0.57 & 0.3 & 0.33 & 0.37 & 37 & 408 & 3765 & 0.74 \\
\hline
\end{tabular}

Results for $6 \mathrm{MPa}$ gasket loading:

\begin{tabular}{|c|c|c|c|c|c|c|c|c|c|}
\hline No. & $A$ & $B$ & $w_{1}$ & $w_{1}$ & $w_{1}$ & $\tau_{1}$ & $\tau_{1}$ & $\tau_{1}$ & $A / \sigma_{\max }$ \\
\hline \hline 1 & 4.96 & 1.62 & 0.35 & 0.27 & 0.38 & 72.4 & 918 & 11359 & 0.72 \\
2 & 5.17 & 1.64 & 0.35 & 0.29 & 0.36 & 41.7 & 740 & 9902 & 0.72 \\
\hline
\end{tabular}

Results for 13 MPa gasket loading:

\begin{tabular}{|c|c|c|c|c|c|c|c|c|c|}
\hline No. & $A$ & $B$ & $w_{1}$ & $w_{1}$ & $w_{1}$ & $\tau_{1}$ & $\tau_{1}$ & $\tau_{1}$ & $A / \sigma_{\max }$ \\
\hline \hline 1 & 11.36 & 2.34 & 0.41 & 0.26 & 0.33 & 43.9 & 717.5 & 8571 & 0.79 \\
2 & 11.62 & 2.49 & 0.42 & 0.25 & 0.32 & 45.3 & 907 & 11042 & 0.79 \\
3 & 10.7 & 2.31 & 0.41 & 0.27 & 0.33 & 64.3 & 930.3 & 9836 & 0.79 \\
4 & 11.12 & 2.47 & 0.38 & 0.26 & 0.36 & 47.5 & 736.5 & 9137.8 & 0.78 \\
\hline
\end{tabular}


In the tables the last columns show that how many percent the maximal gasket stress decreased after the relaxation process. In the case of the worst (often in engineering) the residual stress is $70 \%$ of the maximal gasket stress. If this value does not reach the minimal stress of the gasket, leaking may happen.

\section{Conclusion}

The presented calculation and measuring method is suitable to describe the viscoelastic type gasket time-stress function and determine the residual gasket stress on account of the stress relaxation process. In the future the effects of the re-loading for the relaxation properties will be investigated.

\section{REFERENCES}

1. G. SzePESI and M. OrtutAy: Mathematical model of gas leaking. GÉP, 10-11, (2004), 126-131.

2. K.D. Papoulia, V.P. Panoskaltsis: and I. Korovajchuk: A class of models of 3D finite viscoelasticity School of Civil and Environmental Engineering, Cornell University, Ithaca, USA.

3. A. Galántai and A. Jeney: Numerical Methods Miskolc, University Press, Miskolc, 1998. (in Hungarian)

4. M.A. Del Nobile, S.Chillo, A. Mentana and A. Baiano: Use of the generalized Maxwell model for describing the stress relaxation behavior of solid-like foods. Journal of Food Engineering, 78, (2007), 978-983. (1998)

5. J. A. GonzÁlez and R. AbASCAL: Linear viscoelastic boundary element formulation for steady state moving loads Engineering Analysis with Boundary Elements, 28(7) (2004), 815-823. 